\title{
Causes of HIV and AIDS Stigmatization in Nepal: a Proposed Model to Reduce Stigma
}

\author{
Dave Beine \\ SIL International \\ 2329 E. $46^{\text {th }}$ Ave, Spokane, WA 99223, USA \\ Tel: 1-509-228-8973Ｅ-mail: david_beine@sil.org
}

Received: April 21, 2011 Accepted: May 16, 2011 doi:10.5539/gjhs.v3n2p3

\begin{abstract}
Stigma related to HIV and AIDS in Nepal is an issue that requires better understanding. Some prevention efforts, because of lack of cultural understanding, have paradoxically amplified the problem they were intended to reduce. In this paper I set out to identify the causes of stigma related to HIV and AIDS in Nepal and propose a prevention model that makes use of culture to reduce this more effectively.
\end{abstract}

Keywords: Stigma related to HIV and AIDS, Cultural models, AIDS awareness, HIV prevention models

\section{Introduction}

In the seminal work on stigma, sociologist Erving Goffman (1963) defined stigma as an "attribute that is deeply discrediting" and that reduces its bearer "from a whole and usual person to a tainted, discounted one" (p.3). AVERT (2010), the international AIDS charity, defines AIDS-related stigma and discrimination as "prejudice, negative attitudes, abuse and maltreatment directed at people living with HIV and AIDS (Note 1). They can result in being shunned by family, peers and the wider community; poor treatment in healthcare and education settings; an erosion of rights; psychological damage; and can negatively affect the success of HIV testing and treatment" (Introduction section, note 1).

Many scholars working in the area of HIV and AIDS in Nepal have identified stigma as a serious problem needing more understanding and attention (Beine, 2001; Beine, 2003; Jha \& Madison, 2009; Mahat \& Eller, 2009; Nepal \& Ross, 2010; Silverman et al., 2007; Sing et al., 2005: USAID, 2004; Wasti et al., 2009). In a paradoxical twist, several authors now even suggest that stigma related to HIV and AIDS has actually been amplified by the very prevention models that were implemented to reduce it (Beine, 2001; Beine, 2003; Nepal \& Ross, 2010; Wasti, 2009). As a result of this paradox, many are now recognizing the need to take culture seriously and are advocating for the use of culturally informed prevention models (USAID, 2004; Wasti, 2009; Poudel et. al., 2005). In this paper I set out to identify the causes of stigma related to HIV and AIDS in Nepal and propose a prevention model that makes use of culture to reduce stigma related to HIV and AIDS more effectively.

\section{The causes of and responses to stigma in Nepal}

Recently I read an article which set out to explore the sources, forms and impact of HIV and AIDS stigmatization in Nepal. The authors were also interested in identifying what they term the "mechanism" that creates stigmatization toward people living with HIV and AIDS (PLWHAs). It was intriguing to read: "This study confirms the findings of Beine (2002) who suggested that AIDS as a result of bad karma is a key theme with regard to HIV stigma in Nepal. And the concept of 'hate' as Beine described seems to be a current phenomenon as well. This study also confirms Beine's speculation about the role of the media and health care providers in establishing negative elements such as fear and death within the realm of HIV and AIDS” (Nepal \& Ross, 2010, p. 28).

Likewise, I was encouraged to read that the study's findings "closely tie" with elements of fear and blame that I had identified in my earlier research (ibid, p. 28). Regarding the latter, the authors conclude that their findings "seem to be in conformity with the findings of Beine (2002) who suggested that blame is a key element in defining the understanding of HIV in Nepal” (ibid, p. 29). I was glad to see my earlier hypothesis tested and validated as it relates to the emerging cultural model of HIV and AIDS in Nepal and its impact upon HIV and 
AIDS-related stigma.

Where I differ with the authors of this recent study, however, is in their claim that this element of fear that underlies stigmatization is "triggered by lack of proper knowledge about modes of transmission of infection" (ibid, p. 28). I would argue that it is not principally a lack of knowledge, but rather the instantiation of culturally-based cognitive schemata at work here. As an example, in a recent study, nurses with good knowledge of modes of transmission expressed negative attitudes (stigma) toward PLWHAs, similar to nurses with less knowledge about modes of transmission (Mahat \& Eller, 2009). I found the same true with many doctors back in the late 1990s. They had an adequate understanding (i.e. knowledge) of modes of transmission but a surprising number acted instead on the wider cultural model which produced further stigma (Beine, 2003, p. 252). I find it more likely that stigmatization is principally triggered by the underlying biologically-based cognitive schema that I have identified elsewhere as the "survival schema" (Beine, 2002, p. 304; Beine, 2003, p. 275). Human beings everywhere tend to understand death (and therefore fatal illness) as a kind of "final trump card," and consequently, we act (for the sake of maximal protection) often on our protective instincts rather than on our knowledge. This biological "survival schema" is, in turn, embedded in higher level Nepali cultural schemata (which are reflected linguistically through the Nepali language). For example, the "randi" (sex as taboo), "grena" (despise warranted toward those considered "randi”), and "chichi durdur" (isolation warranted toward those with transmittable disease) schemata create fear, hate and blame (i.e. stigma) as three of the main constituents of the wider cultural model of HIV and AIDS. Interestingly, it is the interplay with traditional illness categories of "sarawa" (transmittable), "untim" (fatal), and "yon rog" (sexual disease) that instantiate these stigmatizing schemata, and it was our western prevention messages that first framed HIV and AIDS in this light (Beine, 2003). So, although these authors identify lack of knowledge as the "mechanism" which creates stigma toward PLWHAs, I find it more likely that the instantiation of culturally-based cognitive schemata, paradoxically the result of the prevention messages we framed and sent, is the mechanism of stigmatization related to HIV and AIDS in Nepal.

As I have noted elsewhere (Beine, 2003, p. 96), “awareness building” has been the primary strategy in Nepal's HIV and AIDS prevention programs, and this is certainly an essential element in HIV prevention. Without awareness building, stigma (acting only on the cultural model) is guaranteed. And it certainly must be acknowledged that through awareness building those with adequate knowledge can choose to act on their newly acquired knowledge rather than upon the cultural model (which some are doing). However, although awareness building can increase knowledge, there is no guarantee that it will reduce stigma (because lack of education is not the principal trigger). And as I have pointed out elsewhere (Beine, 2002; Beine, 2003), and other authors have confirmed (Nepal \& Ross, 2010; Wasti, 2009), the resident elements of our "awareness building” messages can, if we are not careful, actually reinforce and strengthen stigma (by instantiating existing cultural schemata) rather than reducing it. My point here is that knowledge (i.e. awareness building) is essential, but alone it is not enough. If we are to be successful at changing discriminatory practices against PLWHAs in Nepal we must address the cultural models (and underlying biological cognitive schemata) of HIV and AIDS rather than just providing "the facts" in our awareness building campaigns. An understanding of these cultural influences must undergird our efforts and result in culturally-appropriate education materials and methods that incorporate these features if we are to succeed in reducing stigma associated with HIV and AIDS in Nepal (Note 2).

\section{Using culture to reduce stigma}

Recently there has been a growing awareness of the importance of understanding and employing culture in HIV prevention models. Some are even beginning to question the effectiveness of the "standard fare" prevention approaches (i.e. "open discussion," condom as the answer to all, etc.) in the Nepali cultural context and are beginning to advocate for approaches that take culture seriously (and use it) in the equation (Beine, 2003; Wasti, 2009; Pokhrel, 2008). In the late 1990s I set out to define a cultural model of HIV and AIDS in Nepal. Based on the findings of an ethno-medical study conducted in Gorkha District, that sought to understand how rural villagers conceive of HIV and AIDS (Beine, 2001), and on a narrative analysis project conducted among PLWHAs in Kathmandu, that sought to know how they understood their illness, I proposed a cultural model of HIV and AIDS (Beine, 2003). I suggested that because of resident elements (cognitive schemata) of this cultural model, the prevention strategies were actually contributing to the growth of the problem (and stigma) rather than reducing it.

My conclusions from these studies led me to suggest 1) that "the content of HIV and AIDS messages should be different;" prevention planners should "stop associating AIDS so directly with sex in the public prevention campaign materials," 2) that campaigns targeting the general public should move away from first focusing on the fatal (untim) and infectious (sarawa) nature of HIV and AIDS (because of the stigmatizing schemata this 
instantiates), 3) that prevention planners should build upon other resident cultural schemata (such as the cultural ideas of sexual responsibility, avoiding "bad actions," and doing "good works" to build one's karma, etc.) to target PLWHAs, 4) that appropriate linguistic forms should be used (e.g. simple Nepali, local languages where appropriate, and the appropriate Nepali health-related schema of "eating" rather than the corresponding western "war" schema), and 5) that appropriate cultural forms (e.g. songs, drama, etc.) should be employed to convey these HIV prevention messages. Doing so, I conjectured, would also reduce HIV-related stigma.

I am happy to see some of my earlier recommendations emerging in recognizable forms in recent HIV and AIDS-prevention plans. Pokhrel et al. (2008, p. 206) in proposing a model of HIV prevention targeted for rural youth, suggest "avoiding direct references to sex and sexual organs" in public messages about HIV and AIDS. Further, they suggest dividing adolescents along gender, age, and even caste lines when needing to address the more sensitive topics. This latter suggestion wonderfully answers a troubling paradox I was left with in my research (particularly as it relates to adolescents). I was particularly troubled by the real need to adequately educate adolescents before the onset of sexual activity (in order to protect them), and yet the realization that to do so through the use of explicit sexual materials in this cultural context would instantiate the "randi" schema, further reinforcing stigma. The suggestion of separating audiences for these sensitive discussions would seemingly adequately address this concern.

Others have likewise made use of culture--applying sexually neutral messages that use culturally-appropriate containers (i.e. traditional entertainment approaches)--to send stigma reducing public messages about HIV and AIDS. For instance, one current radio program (which targets migrant men working abroad, but to which everyone can listen) includes linguistically and culturally appropriate messages from family members, such as "I have heard about HIV; be careful about it to save our lives," and "Brothers who are in India, come home with money, not with AIDS" (Desh Pardesh, 2010, At home, note 4). Another radio program integrates traditional entertainment forms (e.g. drama and songs) to send culturally-appropriate "general audience" messages about HIV and AIDS which are stigma reducing rather than stigma re-enforcing (Family Health International, 2010). In a unique program that uses traditional Gandhaba singers to disseminate HIV and AIDS information, it appears that "information regarding sexual issues are more acceptable" through this format than via modern media (Dahal, 2008, p. S22). This matches an observation I made in the first chapter of my book when I wrote about being told, "If you want to know the depth of a Nepali's emotion about a certain experience ask them to write a song about it" (Beine 2003, p. 17). It appears that, for whatever reason, traditional forms of entertainment may be more appropriate venues for transmitting culturally-sensitive or taboo information. And some are capitalizing on this fact in creative ways to further reduce HIV and AIDS-related stigma.

Regarding language and culture, I earlier identified various linguistic issues that are impacting HIV and AIDS education campaigns in Nepal (Beine, 2002; Beine, 2003). As a practical example, I found, while working in Gorkha District, that some understood AIDS as a brand of beer. AIDS advertisements had begun to be aired immediately after the news on radio Nepal where the beer advertisements had once been. Because the advertisements were in the high formal language, because the people spoke village Nepali rather than formal Nepali, and because the adds used a foreign illness schema (different from the "eating" schema they use to talk about illness), people could make no sense of the advertisements and concluded that AIDS must be a brand of beer, since the AIDS-prevention advertisements appeared where beer adds had once been (Note 3). This produced a significant misunderstanding about HIV and AIDS.

Pokhrel et al. (2008, p. 203) further address what they term the "linguistic problem" inherent in current HIV prevention materials in Nepal, suggesting that transliteration of English acronyms (e.g. HIV and AIDS)--and their medically technical meanings - are problematic. These technical terms are put into high-level Sanskrit-based Nepali (thulo bhasa), often resulting in non-understanding and misconceptions for rural people. And, as they note, most materials are adapted (including form) from resource materials written in English, which removes it even another level from easy understanding. According to SIL International (2010) only 41\% of the country's population speaks Nepali as their mother tongue, which may further explain why understanding of these messages in thulo bhasa Nepali could be problematic for a majority of the population. Likewise, as a linguist, I have found that many rural "mother tongue" Nepali speakers actually speak forms of "gauli" (village Nepali) rather than formal Nepali. Even these would have trouble understanding the high literary language that is used throughout these materials.

Using our understanding of cultural and linguistic factors I would suggest 1) that all prevention messages be translated into simple understandable Nepali, 2) that all prevention messages use the "eating" illness schema (or other regionally appropriate illness schemata) rather than the western war metaphor, and 3) that we provide materials in mother tongue languages if Nepali is not the mother tongue. 


\section{The suggested model}

Below I offer a comprehensive HIV and AIDS prevention model for consideration which employs many of the recommendations made above and which may be useful for lowering stigma related to HIV and AIDS in Nepal. First, I would suggest that, due to the various linguistic and cultural factors noted above, a "one-size-fits-all" approach (which has been a major strategy thus far) will not work well in Nepal. Using such an approach has the danger of producing messages that actually increase stigma rather than reducing it. For this reason I would suggest targeting different audiences with different messages and even developing "general public" and "group specific" messages for use among various sub-groups.

\subsection{General audience messages}

Because of the "randi" association noted above, I believe it is imperative to stop associating HIV and AIDS so directly with sex in wider audience presentations about HIV and AIDS. To do so only reinforces the "randi" schema, further stigmatizing PLWHAs. Of course we cannot neglect the association of HIV and AIDS with its major vectors, but great care should be taken in doing this, so as not to instantiate further stigmatizing schemata. I suggest, for the acceptance of both speaker and hearer, that whenever possible, local euphemisms and sexually neutral language (such as "avoiding bad actions") be used (again, I think that the potential dangers of doing this are less than the certain limitations of speaking directly as noted above). Of course, people need accurate information about how HIV spreads and how to avoid it (which requires sexually explicit information), but I suggest that when this is done, it be done directly only in the sub-group (gender/age/caste) divisions recommended above.

In regard to campaigns targeting the general public, I suggest moving away from focusing on the fatal (untim) and infectious (sarawa) nature of HIV and AIDS (because doing so will only further stigmatize PLWHAs) and focusing instead on HIV and AIDS as our problem, building on the resident sense of community in Nepali culture (Note 4). Further, these campaigns could highlight structural drivers, such as poverty, which lead people to out migrate. Focusing on these issues is more likely to encourage compassion toward PLWHAs rather than further stigma as the current sexual messages instantiate. Likewise, I suggest building on the resident Nepali Hindu and Buddhist (the main faiths of the region) moral obligation schemata, that stress the religious obligation to love those infected (e.g. doing good works toward the vulnerable in order to build karma), within this general audience model.

Finally, in general audience messages, I suggest that appropriate linguistic forms (e.g. simple Nepali and other local languages where appropriate), the appropriate Nepali health-related schema (e.g. "eating" rather than the corresponding western "war" schema), and appropriate cultural forms (e.g. songs, drama, etc.) be employed to convey these HIV prevention messages. Above all, I suggest that whatever is promoted among the general audience be done in such a way so as not to instantiate the randi-grena schemata (which will further stigmatize PLWHAs).

\subsection{Highest risk groups}

I suggest that much of what is currently being done to target "high-risk" groups in Nepal is appropriate and seemingly effective, but that some modification is warranted. In the AIDS literature for Nepal, the highest risk groups are usually identified as injecting drug users (IDUs), commercial sex workers (CSWs), and migrant workers. For the first two sub-groups (IDUs and CSWs) current methods (and messages) seem appropriate and mostly effective, so not much change is needed in methods or form of the message (although there may be some question about illness schema being employed, but this is more a rural versus urban or education issue which will be discussed later). Regarding these first two sub-groups (IDUs and CSWs), the concerns about using explicit drug and sex related materials, respectively, would be irrelevant since they are already practicing culturally stigmatizing behaviors that are considered "bad" (Note 5). Likewise, it is imperative that these groups get accurate and complete information about how to protect against HIV since they are involved in high-risk practices. Those working in international HIV and AIDS prevention currently endorse the ABC approach as the best evidence-based effective model of prevention (Note 6). The A (abstinence) and B (be faithful) messages would be appropriate for IDUs (to delay the onset of sexual activity and encourage partner reduction) but would be pointless for CSWs who derive their income from sex. However, a focus within these two groups upon "saving others" (through appropriate precautions) is a familiar cultural schema which could be productively built upon within this group.

Because of the highly stigmatized nature of sex and drugs in the Nepali culture, however, I would suggest the use of internal networks for education rather than public mass media campaigns for disseminating any explicit information targeted for these groups. Broadcasting explicit images or use of sexually explicit language, via the 
mass media will further stigmatize this group (because of their association with "randi" behaviors) among other members of the public who are seeing or hearing these messages. We also need to be certain that these two groups are made aware of (and have access to) counseling and ART services.

Although prevalence rates are highest among IDUs and CSWs, migrant laborers and their spouses make up almost 62\% percent of the total estimated HIV infections in the country (USAID 2010, Introduction section, note 2). For this reason I would consider them a specialized high-risk target group that warrants special attention as well. Regarding migrant men and their wives, I would suggest that many of the current prevention approaches are effective. Among the more successful I have observed are radio programs, such as those noted above, which target migrant men and their families and which integrate traditional forms of entertainment, such as drama, comedy and songs, in linguistically and culturally-appropriate forms. These are broadcast abroad and in Western Nepal where out-migration rates are highest. These programs now make use of the "avoiding direct references to sex" idea when they broadcast audio letters from wives and families to their men abroad. Letters from family members to migrant men have also been another successful venue for disseminating prevention messages. Any messages which are in view (or hearing) of the wider public, however, should conform to the explicit language concerns noted above. Perhaps a better venue for disseminating the more necessary explicit information to migrant men would be through the large number of employment agencies that send these men abroad, as well as via visa officers at entry/exit points who process their papers.

The wives of these migrant men must also be targeted in the villages. Beyond the general audience program suggestions above (which would be relevant for them since these women are members of the wider public as well), wives could be encouraged to join a "wives of migrant laborers" group. These specialized women's groups would be a better place to share the more graphic and potentially stigmatizing information. We must provide more relevant information within these groups so that women can protect themselves, but we need to do so very carefully, in a way that does not offend their cultural sensitivities (perhaps using euphemistic language wherever possible). Perhaps being sensitive to the language and cultural issues would ensure that our message is better accepted. The reality is that rural women in Nepal have few sexual rights. We need to continue to work against this negative aspect of culture, but work within the cultural realities. Above all we need to provide counseling options for these women along with HIV education.

\subsection{People Living with HIV and AIDS (PLWHAs)}

Finally, culture is also a factor when working with PLWHAs. In working with PLWHAs, I would suggest that prevention planners build upon resident cultural schemas (such as the cultural ideas of sexual responsibility, avoiding "bad actions," and doing "good works" to build one’s karma, etc.). It would be beneficial to build these ideas and ways of talking about HIV and AIDS into counseling and treatment protocols used with PLWHAs. Above all, we need to remember that beyond being a member of a specific sub-group, each individual is also a member of the wider culture and is subject to the wider cultural model of HIV and AIDS. For this reason it behooves us to understand the wider cultural model and to act and communicate in ways that reduce stigma rather than increasing it.

\section{Conclusions}

Pokhrel et al. (2008) have commented, "Currently not much research seems to have been done on the development of HIV/AIDS prevention programs that have been implemented in Nepal. To better understand the process of adapting HIV/AIDS prevention programs to the Nepalese context, it is necessary that the involved organizations publish and disseminate their experience with program development (p.208). In this paper I set out to identify the causes of stigma related to HIV and AIDS in Nepal and to propose a prevention model that makes use of culture to help reduce stigma related to HIV and AIDS more effectively. This is certainly not a "perfect" model, but rather ideas that need to be further tested for efficacy. I am currently involved in implementing many of these ideas in the Palpa District Community Health Program (associated with Tansen Mission Hospital). I hope that others will do the same and share the information of success or failure so that together we might be able to better beat HIV and AIDS-related discrimination in Nepal in the future.

\section{References}

AVERT. (2010). Stigma, Discrimination and Attitudes to HIV \& AIDS. [Online] Available: http://www.avert.org/hiv-aids-stigma.htm (May 22, 2010)

Baral, S. C., Karki, D. K., \& Newell, J. N. (2007). Causes of Stigma and Discrimination Associated with Tuberculosis in Nepal: a Qualitative Study. BMC Public Health, 7, 211. http://dx.doi.org/10.1186/1471-2458-7-211 
Beine, D. (2001). Saano Dumre Revisited: Changing Models of Illness in a Village of Central Nepal. Contributions to Nepalese Studies, 28 (2), 155-185.

Beine, D. (2002). HIV/AIDS in Nepal: The Making of a Cultural Model. Contributions to Nepalese Studies, 29 (2), 275-310.

Beine, D. (2003). Ensnared by AIDS: Cultural Contexts of HIV/AIDS in Nepal. Kathmandu, Nepal: Mandala Book Point.

Dahal, S. (2008). HIV/AIDS Prevention and Reproductive Health Program Junior/Youth Red Cross Department, Nepal Red Cross Society. International Journal of Infectious Diseases, 12 (Supplement 2).

Desh, Pardesh. (2010). Program Summary. [Online] Available: https://www.comminit.com/en/node/ 308167/347 (May 22, 2010)

Family Health International. (2010). Ek Apas Ka Kura. [Online] Available: http://www.fhi.org/en/ CountryProfiles/Nepal/res_radio.htm (May 22, 2010)

Goffman, E. (1963). Stigma: Notes on the Management of Spoiled Identity. Englewood Cliffs, NJ: Prentice Hall (p.3).

Jha, C. K. \& Madison, J. (2009). Disparity in Health Care: HIV, Stigma, and Marginalization in Nepal. Journal International AIDS Society, 12, 16. http://dx.doi.org/10.1186/1758-2652-12-16

Mahat, G. \& Eller, L. S. (2009). HIV and Universal Precautions: Knowledge and Attitudes of Nepalese Nursing Students. Journal of Advanced Nursing, 65 (9), 1907-1915. http://dx.doi.org/10.1111/j.1365-2648.2009.05070.x

NCASC. (2010). National Center for AIDS and STD Control: Government of Nepal, Ministry of Health and Population. Cumulative HIV and AIDS Situation in Nepal as of October, 2009. [Online] Available: http://www.ncasc.gov.np/pics/category/files/1259600551.pdf (May 25, 2010)

Nepal, V. P. \& Ross, M. W. (2010). Issues Related to HIV Stigma in Nepal. International Journal of Sexual Health, 22, 20-31. http://dx.doi.org/10.1080/19317610903393142

Pokhrel, P., Regmi, S., \& Piedade, E. (2008). HIV/AIDS Prevention in the Nepalese Context. Evaluation \& the Health Professions, 31 (2), 198-210. http://dx.doi.org/10.1177/0163278708315924

Poudel, K. C., Jimba, M., Joshi, A., Poudel-Tandukar, K., Sharma, M., \& Wakai, S. (2005). Retention and Effectiveness of HIV/AIDS Training of Traditional Healers in Far Western Nepal. Tropical Medicine \& International Health, 10 (7), 640-646. http://dx.doi.org/10.1111/j.1365-3156.2005.01443.x

SIL International. (2010). Ethnologue: Languages of the World, $16^{\text {th }}$ ed. [Online] Available: http://www.ethnologue.com/show_language.asp?code=nep (May 22, 2010)

Silverman, J. G., Decker, M. R., Gupta, J., Maheshwari, A., Willis, B. M., \& Raj, A. (2007). HIV Prevalence and Predictors of Infection in Sex-trafficked Nepalese Girls and Women. JAMA. 298, 536-542. http://dx.doi.org/10.1001/jama.298.5.536

Sing, S. K., Manandhar, N., Prasai, M., Patowary, S., \& Krishna, G. (2005). An Awareness Study of HIV/AIDS among Adolescent Students of Chitwan District, Nepal. Journal of the Institute of Medicine, 27 (3). http://dx.doi.org/10.3126/joim.v27i3.410

Wasti, S. P., Simkhada, P., Randall, J., \& VanTeijlingen, E. (2009). Issues and Challenges of HIV/AIDS Prevention and Treatment Programme in Nepal. Global Journal of Health Science, 1 (2), 62-72.

USAID. (2004). Assessment of youth reproductive health/HIV programs in Nepal. Kathmandu, Nepal: USAID.

USAID. (2010). HIV/AIDS health profile for Nepal, October, 2010. [Online] Available: http://www.usaid.gov/our_work/global_health/aids/Countries/asia/nepal_profile.pdf (May 25, 2011)

\section{Notes}

Note 1. HIV (human immunodeficiency virus) is an infective agent, while AIDS (acquired immunodeficiency syndrome) is a condition of the body (usually characterized by a collection of symptoms such as long-term fevers, swollen glands, weakness, and weight loss). Unless stemmed by anti-retroviral therapy (ART), HIV usually precipitates AIDS, a condition which progressively weakens the human.

immune system by allowing life-threatening opportunistic infections to ultimately overwhelm the body. This weakening condition is usually not considered "AIDS" until the CD4 count is less than 200 cells per $\mu \mathrm{L}$ of blood. 
Note 2. Interestingly Baral et al. (2007) identify “fear of perceived risk of infection,” “perceived links between TB and disreputable behavior," and "perceptions that TB was a divine punishment” among the four major causes of stigma and discrimination associated with tuberculosis in Nepal. These same associations are made in regard to HIV and AIDS as I have illustrated elsewhere (Beine, 2002; Beine, 2003). Given my earlier research, this is no surprise, since TB and HIV/AIDS are culturally categorized together as two of the main constituent members of the class of illness known as "sarawa rogharu” (transmittable illness). Therefore, instantiating a cognitive schema that produces like understanding, it is no surprise to see similar associations (and stigmatizing behavior) being made toward two members of the same class of illness.

Note 3. The use of a foreign western ("warfare”) illness schemata instead of the locally understood "eating” schemata for illness lead to confusion about the meanings of HIV and AIDS in rural Nepal. For a description of this process, along with a discussion of the various other potential problems related to what I termed "the language of AIDS," see Beine (2003).

Note 4. A good example of this (that employs both "war" and "eating” schemata for illustrative purposes) is, "Together we can (b) eat HIV and AIDS!"

Note 5. Although still categorized as "bad” I have demonstrated how drug use is considered less bad than sexual sin (Beine, 2003).

Note 6. For a good review of ABC (its current meaning and controversies). [Online] Available: http://www.avert.org/ abc-hiv.htm 